\title{
User perception of medical service robots in hospital wards: a cross-sectional study
}

\author{
Jung Hwan Lee, Jae Meen Lee, Jaehyun Hwang, Joo Young Park, Mijeong Kim, Dong Hwan Kim, Jae Il Lee, \\ Kyoung Hyup Nam, In Ho Han
}

Department of Neurosurgery and Medical Research Institute, Pusan National University Hospital, Busan, Korea

Background: Recently, there have been various developments in medical service robots (MSRs). However, few studies have examined the perceptions of those who use it. The purpose of this study is to identify user perceptions of MSRs.

Methods: We conducted a survey of 320 patients, doctors, and nurses. The contents of the survey were organized as follows: external appearances, perceptions, expected utilization, possible safety accidents, and awareness of their responsibilities. Statistical analyses were performed using $t$-test, chi-square test, and analysis of variance.

Results: The most preferred appearance was the animal type, with a screen. The overall average score of positive questions was $3.64 \pm 0.98$ of 5 points and that of negative questions was $3.24 \pm 0.99$. Thus, the results revealed that the participants had positive perceptions of MSR. The overall average of all expected utilization was $4.05 \pm 0.84$. The most expected utilization was to guide hospital facilities. The most worrisome accident was exposure to personal information. Moreover, participants thought that the overall responsibility of the robot user (hospital) was greater than that of the robot manufacturer in the case of safety accidents.

Conclusion: The perceptions of MSRs used in hospital wards were positive, and the overall expected utilization was high. It is necessary to recognize safety accidents for such robots, and sufficient attention is required when developing and manufacturing robots.

Keywords: Hospitals; Perception; Robotics; Surveys and questionnaires

\section{Introduction}

The fourth industrial revolution, represented by big data, the Internet of Things, artificial intelligence, and robotics, is underway worldwide [1]. Numerous studies have examined the development of various robots to replace human tasks [2]. There have also been many studies related to robots in the medical field. These include surgical robots, rehabilitation robots, nursing assistant robots, and hospital logistics robots [3]. Among these robots, surgical robots have been actively used [4]. However, with the exception of some telemedicine robots, there are few cases in which ro- bots can care or monitor the patient in a real hospital. Nevertheless, advances in robot technology continue to increase interest in medical service robots (MSRs) that can replace or reduce medical and nursing work in hospitals. However, actual medical service development should begin with a sufficient understanding of the actual needs in the medical field and possible problems. Although there have been perception surveys for some nurses so far, they have been limited to care robots $[3,5]$. Those who use medical robots in hospital wards include nurses, patients who receive medical services, and doctors. The perceptions of MSR users are also very important, but there are no multi-dimensional perception surveys

Received: July 19, 2021 • Revised: August 8, 2021 - Accepted: August 21, 2021

Corresponding author: In Ho Han, MD, PhD

Department of Neurosurgery, Pusan National University Hospital, 179 Gudeok-ro, Seo-gu, Busan 49241, Korea

Tel: +82-51-240-7257•Fax: +82-51-244-0282•E-mail: farlateral@pusan.ac.kr

Copyright (c) 2022 Yeungnam University College of Medicine, Yeungnam University Institute of Medical Science

This is an Open Access article distributed under the terms of the Creative Commons Attribution Non-Commercial License (http://creativecommons.org/licenses/by-nc/4.0/) which permits unrestricted non-commercial use, distribution, and reproduction in any medium, provided the original work is properly cited. 
on MSR that include doctors and patients.

Therefore, the purpose of this study was to investigate user perceptions, needs, and possible problems for MSRs before developing robots that assist or replace treatment and nursing for patients in hospital wards. It also aims to provide important information for robot developers who wish to develop medical robots by evaluating user perceptions.

\section{Methods}

Ethical statements: With the approval of the Institutional Review Board (IRB) of Pusan National University Hospital (IRB No: 1909-016-083), a survey was conducted with doctors, nurses, and patients in the ward. All participants provided written informed consent.

\section{Survey participants}

The number of participants was calculated using the PASS 11 (NCSS, LLC., East Kaysville, UT, USA). The preference for MSRs was expected to be over $60 \%$. If the width of the $95 \%$ confidence interval was within $12 \%$ and an error of $6 \%$ was allowed, the required sample size was confirmed to be 271 . Considering the rate of dropouts such as abandonment and omission as $20 \%$, a total of 325 copies were distributed. Finally, 320 copies were collected (recovery rate, $98.5 \%$ ), which were used for the analysis.

\section{Questionnaire}

The MSR was introduced first as follows: "We are going to conduct a perception survey on MSRs for inpatients. The robot will help the staff (doctors and nurses). Autonomous driving is possible, and information delivery and education will be possible through the screen. Partial dialog (communication through speech) will be possible, and images will be collected and analyzed through depth cameras."

The questionnaire was largely composed of four items: (1) preferred external appearance, (2) perception, (3) expected utilization, (4) predicted safety accidents and their responsibilities. The preferred external appearance list is shown in Fig. 1. The questions associated with the perceptions of MSR are presented in Table 1. Questions associated with perceptions were created by mixing positive and negative questions, and the responses were answered on a Likert 5-point scale from "not at all (1 point)" to "strongly agree (5 points)." The expected utilization of MSRs is listed in Table 2. The responses were answered on a Likert 5-point scale from "not at all important (1 point)" to "very important (5 points)." In questions associated with possible safety accidents of MSRs and their responsibilities, the answer sheet of the four-or five-choice multiple types was given, and one of them was selected.

\section{Statistical analysis}

All statistical analyses were conducted using IBM SPSS version 22 (IBM Corp., Armonk, NY, USA) and R version 3.6.2 (R Foundation for Statistical Computing, Vienna, Austria). Categorical data were analyzed using Pearson chi-square test. Continuous data were
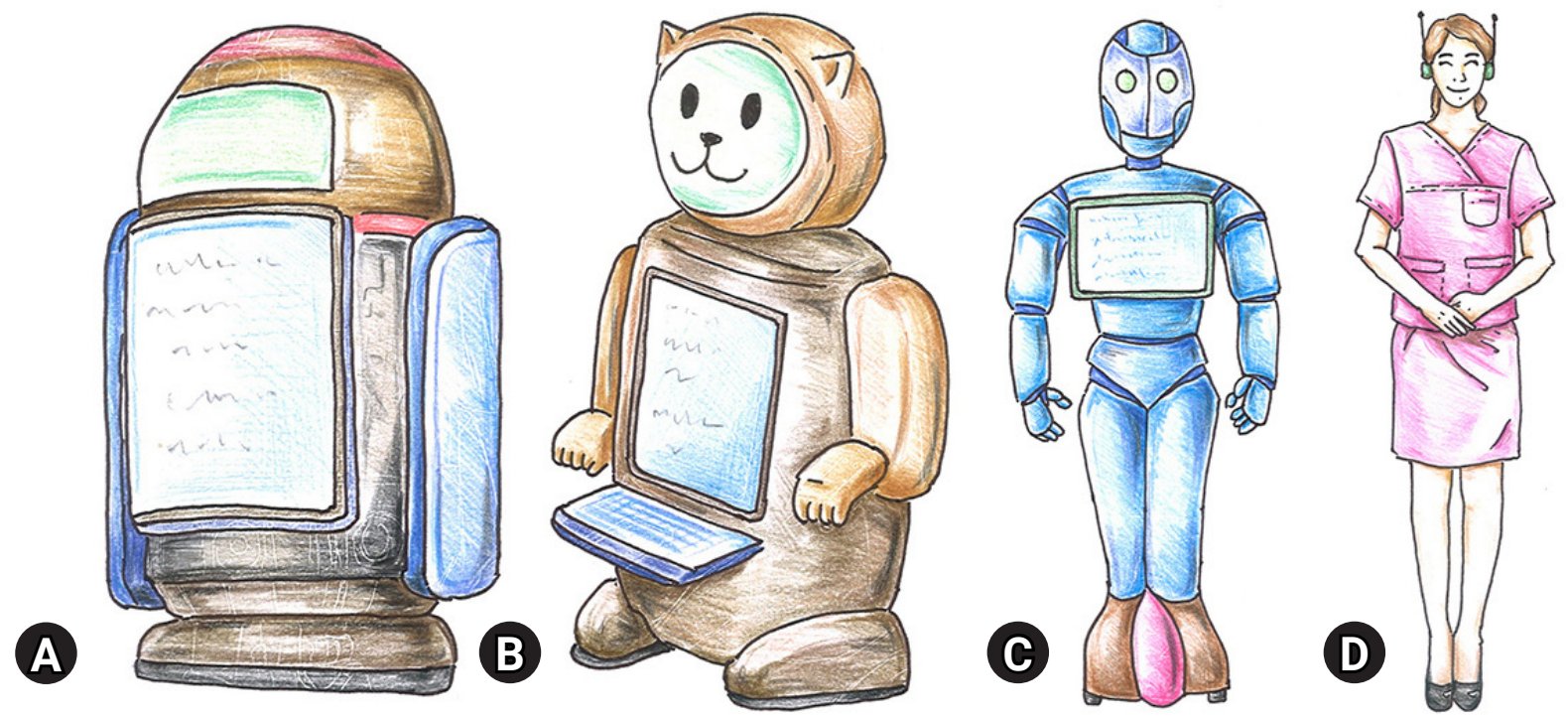

Fig. 1. List of the external appearance of medical service robots. (A) Cylindrical or square type with a screen, (B) animal type with a screen, (C) humanoid (a simplified human structure), and (D) android (very similar to human appearance). 
Table 1. Questionnaire regarding perceptions of medical serviced robots in a hospital

\begin{tabular}{lll}
\hline No. & Category & \\
\hline 001 & Positive & There will be fewer errors in measurement, transferring information, and explanation because it is a machine \\
002 & Positive & There will be less stress that occurs when facing people \\
003 & Positive & Better quality care and hospitalization will be possible by sharing the work of the staff \\
004 & Positive & The conversation function will alleviate the anxiety that occurs during hospitalization \\
005 & Negative & I don't think there are many functions that can replace people, so I don't think I will use it even in wards \\
006 & Negative & There is a possibility that the robot may malfunction \\
007 & Negative & It will be inconvenient to use because I am not good at handling machines \\
008 & Negative & There is a possibility of a safety accident due to autonomous driving \\
009 & Negative & The sensors used by the robot will be less accurate \\
010 & Negative & The communication function used by robots will be less accurate \\
011 & Negative & The information, such as guidance and education provided by robots, will not be of much help in actual situations \\
012 & Positive & The loading of the staff will decrease when the robots share the work \\
013 & Positive & When robots share medical care, patients' satisfaction during hospitalization will be improved \\
014 & Positive & Overall, I agree to deploy robots in wards \\
015 & Positive & Overall, the services provided by robots in wards will be reliable \\
016 & Positive & Overall, the services provided by robots will help patients \\
\hline
\end{tabular}

Table 2. Questionnaire regarding expected utilization of medical service robots

\begin{tabular}{|c|c|}
\hline No. & Service \\
\hline S01 & Checking for the ward environment (temperature, humidity, and airborne dust concentration, etc.) \\
\hline SO2 & Analysis of gait \\
\hline S03 & Instructing rehabilitation methods \\
\hline SO4 & Guiding postoperative posture and range of movement \\
\hline S05 & Checking for paralysis \\
\hline S06 & Calling the ward nurses \\
\hline S07 & Analysis of pain scale \\
\hline S08 & Analysis and improvement of depression/anxiety \\
\hline So9 & Analysis and improvement of stress \\
\hline S10 & Guiding to hospital facilities \\
\hline S11 & Instructing the process of admission and discharge of the hospital \\
\hline S12 & Informing results of imaging and laboratory findings \\
\hline S13 & Informing scheduled inspections \\
\hline S14 & Informing processes and side effects of scheduled surgeries or procedures \\
\hline S15 & Requesting medical certifications \\
\hline S16 & Checking the blood pressure and pulse \\
\hline S17 & Checking the temperature \\
\hline S18 & Checking the respiratory rate \\
\hline S19 & Checking the blood sugar level \\
\hline S20 & Checking the input and output \\
\hline S21 & Checking the amount of remnant intravenous fluid \\
\hline S22 & Guiding to the dietary plan \\
\hline S23 & Management of the drug administration \\
\hline
\end{tabular}

tested using an independent $t$-test and one-way analysis of variance with a post hoc Tukey test. Statistical significance was set at $p<0.05$. Cronbach alpha coefficient was calculated to verify the reliability of the questions associated with perceptions. A scatter plot was constructed to determine the difference in preference for expected utilization between the groups. 


\section{Results}

\section{Characteristics of participants}

A total of 320 participants responded to the survey, and their characteristics are presented in Table 3 . The average age of the respondents was $39 \pm 15$ years.

\section{Preference for the external appearance of medical ser- vice robots}

We presented external images of the MSR (Fig. 1) and chose the most preferred image. B (animal type with screen, 35.0\%) was selected. This was followed by A (cylindrical or square type with a screen, 27.8\%) and D (android [very similar to human appear-

Table 3. Demographic of participants

\begin{tabular}{lcccc}
\hline Characteristic & Patient & Doctor & Nurse & Total \\
\hline Sex & & & & \\
$\quad$ Male & 53 & 58 & 6 & $117(36.6)$ \\
$\quad$ Female & 47 & 46 & 110 & $203(63.4)$ \\
Age group (yr) & & & & \\
$\quad$ Young $(<41)$ & 9 & 76 & 112 & $197(61.6)$ \\
$\quad$ Middle $(\geq 41,<64)$ & 60 & 23 & 4 & $87(27.2)$ \\
$\quad$ Elderly $(\geq 64)$ & 31 & 5 & 0 & $36(11.2)$ \\
Total & $100(31.3)$ & $104(32.5)$ & $116(36.3)$ & $320(100)$ \\
\hline
\end{tabular}

Values are presented as number only or number (\%). ance], 22.4\%). C (humanoid [a simplified human structure], $14.8 \%)$ was the least preferred. The difference in the preference for external appearance according to sex, age group, and occupation classification was not statistically identified.

\section{Perceptions of medical service robots}

Among the questions for the perception of MSRs, the positive questions were Q01-Q04 and Q12-Q16. The overall average score of these questions was $3.64 \pm 0.98$ of 5 points. The negative questions were Q05-Q11 and the overall average score of these questions was $3.24 \pm 0.99$ of 5 points. The value of Cronbach alpha for the applicability to all questions was 0.767 . Cronbach alpha values for the positive and negative questions were 0.826 and 0.735 , respectively. Thus, the overall reliability was acceptable. When the values of the positive and negative questions were compared, the value of the positive questions was statistically significantly higher $(p<0.001)$. The values of each question for perceptions and differences according to occupation classification are listed in Table 4.

\section{Expected utilization of services provided by medical service robots}

The overall average of all expected utilization was $4.05 \pm 0.84$ of 5 points. The value of Cronbach alpha for the applicability for all items was 0.934 . The values of each item for expected utilization and differences according to occupation classification are listed in

Table 4. Statistical analysis of perceptions of medical serviced robots in a hospital

\begin{tabular}{|c|c|c|c|c|c|c|}
\hline Question & Overall & Patient $^{\mathrm{a}}$ & Doctor $^{b}$ & Nurse $^{c}$ & $p$-value & Post hoc analysis \\
\hline 001 & $3.46 \pm 1.05$ & $3.08 \pm 1.12$ & $3.70 \pm 1.01$ & $3.57 \pm 0.96$ & $<0.001$ & $a<b, c$ \\
\hline 002 & $3.83 \pm 1.00$ & $3.49 \pm 1.04$ & $3.98 \pm 0.90$ & $3.97 \pm 1.00$ & 0.001 & $a<b, c$ \\
\hline 004 & $3.22 \pm 0.96$ & $3.45 \pm 0.95$ & $3.02 \pm 1.04$ & $3.19 \pm 0.86$ & 0.009 & $a>b$ \\
\hline 012 & $3.58 \pm 0.88$ & $3.77 \pm 0.88$ & $3.48 \pm 0.82$ & $3.51 \pm 0.91$ & 0.400 & \\
\hline 013 & $3.56 \pm 0.78$ & $3.71 \pm 0.84$ & $3.47 \pm 0.79$ & $3.50 \pm 0.70$ & 0.065 & \\
\hline 015 & $3.62 \pm 0.78$ & $3.74 \pm 0.80$ & $3.56 \pm 0.80$ & $3.57 \pm 0.74$ & 0.195 & \\
\hline 016 & $3.82 \pm 0.69$ & $3.96 \pm 0.64$ & $3.79 \pm 0.70$ & $3.73 \pm 0.71$ & 0.041 & $a>c$ \\
\hline 005 & $2.93 \pm 0.97$ & $2.69 \pm 1.00$ & $2.89 \pm 0.99$ & $3.18 \pm 0.87$ & 0.001 & $a<c$ \\
\hline 006 & $3.84 \pm 0.79$ & $3.65 \pm 0.82$ & $3.81 \pm 0.80$ & $4.04 \pm 0.71$ & 0.001 & $a<c$ \\
\hline 007 & $3.29 \pm 0.99$ & $3.42 \pm 1.01$ & $3.20 \pm 1.02$ & $3.26 \pm 0.94$ & 0.275 & \\
\hline 011 & $2.59 \pm 0.90$ & $2.45 \pm 0.91$ & $2.73 \pm 0.91$ & $2.58 \pm 0.88$ & 0.097 & \\
\hline Total (positive) & $3.64 \pm 0.98$ & $3.68 \pm 0.89$ & $3.61 \pm 0.90$ & $3.64 \pm 0.86$ & 0.255 & \\
\hline Total (negative) & $3.24 \pm 0.99$ & $3.04 \pm 1.04$ & $3.24 \pm 0.97$ & $3.41 \pm 0.93$ & 0.004 & $a<b<c$ \\
\hline
\end{tabular}

Values are presented as mean \pm standard deviation.

Positive questions, 001-004 and 012-016; negative questions, 005-011. 
Table 5. Expected utilization of medical service robots

\begin{tabular}{|c|c|c|c|c|c|c|}
\hline Service & Overall & Patient $^{a}$ & Doctor $^{b}$ & Nurse $^{c}$ & $p$-value & Post hoc analysis \\
\hline S01 & $3.89 \pm 0.85$ & $4.11 \pm 0.67$ & $3.82 \pm 0.97$ & $3.77 \pm 0.86$ & 0.002 & $a>b, c$ \\
\hline S02 & $3.87 \pm 0.98$ & $4.04 \pm 0.86$ & $3.69 \pm 1.12$ & $3.90 \pm 0.91$ & 0.044 & $a>b$ \\
\hline S03 & $4.06 \pm 0.89$ & $4.10 \pm 0.85$ & $3.84 \pm 1.00$ & $4.22 \pm 0.79$ & 0.090 & \\
\hline S04 & $4.14 \pm 0.92$ & $4.17 \pm 0.88$ & $3.83 \pm 1.02$ & $4.39 \pm 0.79$ & $<0.001$ & $b<c$ \\
\hline S05 & $3.98 \pm 1.12$ & $4.22 \pm 0.73$ & $3.58 \pm 1.30$ & $4.15 \pm 1.12$ & $<0.001$ & $a, c>b$ \\
\hline S06 & $4.16 \pm 0.84$ & $4.13 \pm 0.87$ & $4.35 \pm 0.84$ & $4.02 \pm 0.78$ & 0.012 & $b>c$ \\
\hline S07 & $3.98 \pm 0.93$ & $4.15 \pm 0.81$ & $3.79 \pm 1.00$ & $4.00 \pm 0.93$ & 0.019 & $a>b$ \\
\hline S08 & $3.77 \pm 1.11$ & $3.97 \pm 1.23$ & $3.47 \pm 0.99$ & $3.86 \pm 1.05$ & 0.002 & $a>b$ \\
\hline S09 & $3.71 \pm 1.03$ & $3.93 \pm 1.16$ & $3.48 \pm 0.98$ & $3.72 \pm 0.93$ & 0.011 & $a>b$ \\
\hline S10 & $4.44 \pm 0.79$ & $4.26 \pm 0.84$ & $4.46 \pm 0.76$ & $4.57 \pm 0.74$ & 0.160 & \\
\hline S11 & $4.42 \pm 0.84$ & $4.11 \pm 0.85$ & $4.51 \pm 0.86$ & $4.61 \pm 0.75$ & $<0.001$ & $a<c$ \\
\hline S12 & $4.27 \pm 1.01$ & $4.19 \pm 0.71$ & $4.16 \pm 1.19$ & $4.42 \pm 1.04$ & 0.111 & \\
\hline S13 & $4.34 \pm 0.86$ & $4.23 \pm 0.63$ & $4.25 \pm 1.10$ & $4.53 \pm 0.74$ & 0.005 & $a, b<c$ \\
\hline S14 & $4.20 \pm 1.05$ & $4.21 \pm 0.98$ & $3.98 \pm 1.22$ & $4.38 \pm 0.91$ & 0.026 & $b<c$ \\
\hline S15 & $4.28 \pm 0.96$ & $4.14 \pm 0.88$ & $4.25 \pm 1.15$ & $4.44 \pm 0.83$ & 0.35 & \\
\hline S16 & $4.15 \pm 1.02$ & $3.84 \pm 1.13$ & $4.12 \pm 0.91$ & $4.45 \pm 0.93$ & $<0.001$ & $a, b<c$ \\
\hline S17 & $4.16 \pm 1.00$ & $3.82 \pm 1.01$ & $4.12 \pm 0.95$ & $4.48 \pm 0.93$ & $<0.001$ & $a, b<c$ \\
\hline S18 & $4.13 \pm 1.00$ & $3.86 \pm 1.14$ & $4.04 \pm 0.85$ & $4.46 \pm 0.93$ & $<0.001$ & $a, b<c$ \\
\hline S19 & $4.11 \pm 0.96$ & $3.80 \pm 1.02$ & $4.05 \pm 0.83$ & $4.44 \pm 0.94$ & $<0.001$ & $a, b<c$ \\
\hline S20 & $4.16 \pm 1.02$ & $4.15 \pm 1.15$ & $3.98 \pm 0.93$ & $4.32 \pm 0.96$ & 0.031 & $b<c$ \\
\hline S21 & $4.05 \pm 0.91$ & $4.10 \pm 0.83$ & $3.76 \pm 0.99$ & $4.28 \pm 0.84$ & $<0.001$ & $a, c>b$ \\
\hline S22 & $4.04 \pm 0.92$ & $3.99 \pm 0.85$ & $3.82 \pm 1.00$ & $4.29 \pm 0.84$ & 0.001 & $a, b<c$ \\
\hline S23 & $4.07 \pm 0.99$ & $4.12 \pm 1.06$ & $3.77 \pm 1.01$ & $4.29 \pm 0.85$ & $<0.001$ & $a, c>b$ \\
\hline Total & $4.11 \pm 0.63$ & $4.07 \pm 0.58$ & $3.96 \pm 0.61$ & $4.28 \pm 0.65$ & $<0.001$ & $a, b<c$ \\
\hline
\end{tabular}

Values are presented as mean \pm standard deviation.

Table 5. The most expected utilization was to guide hospital facilities. In contrast, the least expected utilization was gait analysis via the MSR. The scatter plots were drawn according to classifications of occupation: patients, doctors, patients, nurses, doctors, and nurses (Fig. 2). In addition to the services presented in the questionnaire, participants' additional suggestions associated with the services provided by MSRs were as follows: transferring specimens, transferring drugs, transferring necessary supplies, managing ward supplies, emergency alerts, measurement of oxygen saturation, and notification associated with clinical work.

\section{Possible safety accidents caused by medical service ro- bots and awareness of their responsibilities}

When an MSR was placed in the ward, the possible safety accidents that were selected as of greatest concern were the transmission of false information and exposure of patient information due to malfunctions (45.5\%). Next, collisions due to autonomous driving (38.1\%), exposure to electromagnetic waves (6.5\%), and fires (6.3\%) were followed. Differences in possible safety accidents according to sex, age groups, and classifications of occupation were not statistically identified. Other opinions included electric shock accidents.

In the case of such accidents, the most frequent answer to the question about the responsibility for the accident was that both the hospital and the robot manufacturing company were equally responsible (34.7\%). This was followed by "the hospitals must be more responsible than the robot manufacturing company" (19.4\%), "the hospitals were entirely responsible" (16.9\%), "the robot manufacturing company must be more responsible than the hospital" (16.6\%), and "the robot manufacturing company was entirely responsible" (12.5\%). No statistical significance was observed for these responses.

The answer most frequently selected as an important factor for the safety of autonomous robots was a low-centered design (33.8\%). This was followed by a soft outer material that can absorb shocks (30.7\%), a height similar to that of a human (15.1\%), and a slow driving speed (13.6\%). Other opinions included the application of collision prevention sensors and the application of robot-only roads. 

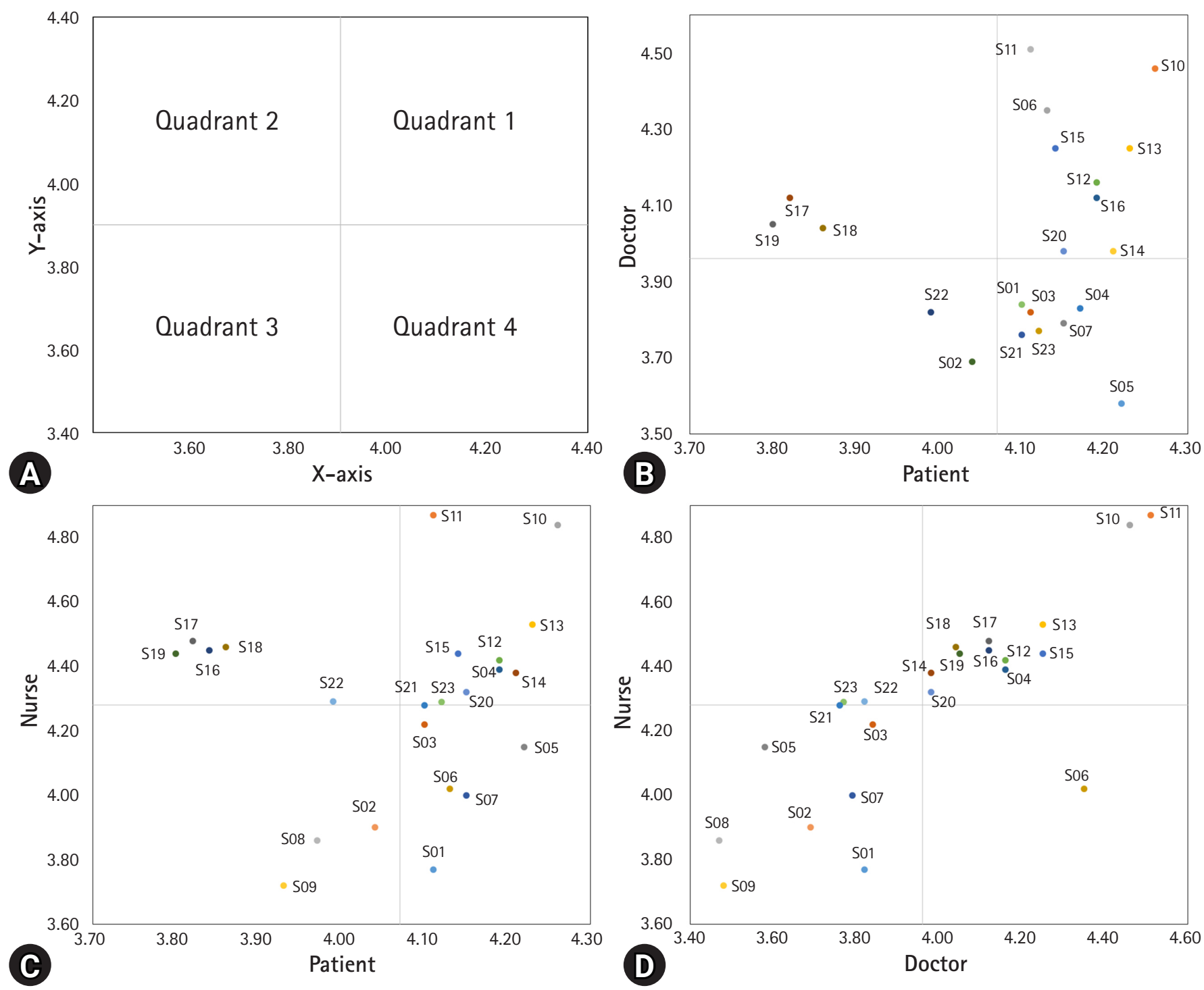

Fig. 2. Scatter plots for expected utilization. (A) Interpretation of a scatter plot. Items in quadrant 1 are preferred for both the x-axis and $y$-axis groups. Items in quadrant 2 are less preferred in the $x$-axis group but are more preferred in the $y$-axis group. Items in quadrant 3 are less preferred for both the $x$-axis and $y$-axis groups. Items in quadrant 4 are more preferred in the $x$-axis group but are less preferred in the $y$-axis group. (B) The scatter plot for the doctor-patient groups. (C) The scatter plot for the nurse-patient groups. (D) The scatter plot for the doctor-nurse groups.

\section{Discussion}

There have been some studies related to the external appearance of social robots to interface with a person [6-8]. Most of them have reported that an animal-like appearance is preferred over a human-like appearance in robots related to healthcare $[6,7]$. In this study, the preference for a non-human-like appearance was higher than that of a human-like appearance. In the case of human-like appearance, social and ethical issues can occur, so caution might be required [7]. Thus, a non-human-like appearance is appropriate. However, in the healthcare field, robots with a human-like appear- ance are preferred [8]. Therefore, additional studies are required to confirm this hypothesis.

Overall, the study participants had positive perceptions of MSR. In the patient group, there were statistically significant positive responses compared to medical staff (doctors and nurses), indicating that the anticipation for MSR was higher. However, even though they were negative questions, some questions exhibited high scores. In particular, scores for robot malfunctions and safety accidents were greater than the median value of the scores for negative questions, indicating concern about this. Among the positive questions, the question with the lowest score was about conversational 
function, and in particular, the doctor group revealed ambivalent perception.

The overall expectation for the utilization of MSR was found to be statistically significantly higher in the nurse group and the lowest in the doctor group. Among the expected utilization of MSR, the service with the highest score was guiding to hospital facilities (S10), and the service with the lowest score was analysis and improvement of stress (S09). The doctor group expected the lowest utilization for analysis and improvement of depression or stress when hospitalized, but the patients expected relatively high efficacy. The patient group expected the highest utilization for guiding to hospital facilities. The services that were judged to show the lowest expected utilization in the patient group were various measurements such as input and output, blood pressure, temperature, respiratory rate, and blood sugar. In contrast, nurses who directly managed these measurements expected relatively high efficacy. In previous surveys related to robots, measurement and monitoring showed the highest preference for nurses, which was similar to our results $[3,5]$. Therefore, it can be seen that nurses prefer to apply MRS to measurement tasks. In the scatterplot analysis, the services that were in the 1st quadrant in all groups (i.e., with relatively high utilization expectations in all groups) were guiding to hospital facilities (S10), instructing the process of admission and discharge of the hospital (S11), informing results of imaging and laboratory findings (S12), informing scheduled inspections (S13), informing processes and side effects of scheduled surgeries or procedures (S14), requesting medical certifications (S15), and checking the input and output (S20). It was mainly related to information delivery to patients or guardians and administrative work processing. In contrast, the service in the third quadrant in all groups, that is, with relatively low utilization expectation in all groups, was S02. There have been many studies related to gait analysis using artificial intelligence [9]. However, it was revealed that expectations were relatively low when applying this function through MSRs.

To use the MSR in hospital wards, it must be able to move to the bed. This was because it was the patient who faced MSR primarily, and the patient was often unable to move out of bed during the acute phase. Thus, our consortium was trying to apply autonomous driving and was concerned about the physical accidents caused by it. However, the most worrisome accident in the survey was exposure to personal information. Therefore, it is necessary to take measures to prevent such accidents when operating an MSR. While the issue of legal responsibility for the occurrence of safety accidents caused by various robots has been actively discussed recently, there has been little discussion about the legal issue of accidents involving robots related to healthcare [10]. Participants in this study thought that the overall responsibility of the robot user (hospital) was greater than that of the robot manufacturer in the case of safety accidents. However, since the judgment of the relevant expert was important in legal matters, more research is needed in this area.

In this study, we investigated the perception associated with MSRs used in hospital wards. The recognition of MSRs used in hospital wards was generally positive, and the overall expected utilization was high. In particular, MSRs were expected to be highly effective in delivering various types of information and measuring the input and output. Furthermore, it is also necessary to recognize safety accidents for such robots, and sufficient attention is required when developing and manufacturing robots.

\section{Notes}

\section{Conflicts of interest}

No potential conflict of interest relevant to this article was reported.

\section{Funding}

This work was supported by the Technology Innovation Program of the Ministry of Trade, Industry \& Energy (MOTIE), funded by the South Korea government (grant number 20000515).

\section{Author contributions}

Conceptualization: JHL, MK, DHK, IHH; Data curation: JHL, JH, JYP, MK, DHK, JIL, KHN; Formal analysis: JML, JIL, IHH; Funding acquisition: MK, DHK, IHH; Methodology: JHL, KHN; Project administration, Resources, Supervision: IHH; Investigation, Software: JHL; Visualization: JML; Validation: MK, DHK; Writing - original draft: JHL, JH, JYP, MK; Writing - review \& editing: JML, DHK, JIL, KHN, IHH.

\section{ORCID}

Jung Hwan Lee, https://orcid.org/0000-0002-1393-7105

Jae Meen Lee, https://orcid.org/0000-0002-5708-1644

Jaehyun Hwang, https://orcid.org/0000-0002-6730-2092

Joo Young Park, https://orcid.org/0000-0001-5646-0899

Mijeong Kim, https://orcid.org/0000-0003-3307-0315

Dong Hwan Kim, https://orcid.org/0000-0001-8982-7917

Jae Il Lee, https://orcid.org/0000-0003-1412-4146

Kyoung Hyup Nam, https://orcid.org/0000-0002-3749-4660

In Ho Han, https://orcid.org/0000-0001-7193-6533

\section{References}

1. Nam KH, Kim DH, Choi BK, Han IH. Internet of things, digi- 
tal biomarker, and artificial intelligence in spine: current and future perspectives. Neurospine 2019;16:705-11.

2. Wang TM, Tao Y, Liu H. Current researches and future development trend of intelligent robot: a review. Int J Autom Compt 2018;15:525-46.

3. Lee JY, Song YA, Jung JY, Kim HJ, Kim BR, Do HK, et al. Nurses' needs for care robots in integrated nursing care services. J Adv Nurs 2018;74:2094-105.

4. Koh DH, Jang WS, Park JW, Ham WS, Han WK, Rha KH, et al. Efficacy and safety of robotic procedures performed using the da Vinci robotic surgical system at a single institute in Korea: experience with 10000 cases. Yonsei Med J 2018;59:975-81.

5. Hong E, Shin S. Nurses' perceptions of care robots in long-term care facilities. J Korean Gerontol Nurs 2019;21:22-32.

6. Lohse M, Hegel F, Swadzba A, Rohlfing K, Wachsmuth S, Wrede B. What can I do for you? Appearance and application of robots. Paper presented at: The 33rd Annual Convention of the
Society for the Study of Artificial Intelligence and the Simulation of Behaviour; 2007 Apr 2-5; Tyne, UK. New York: Curran Associates, Inc.; 2007.p. 121-6

7. Wu YH, Fassert C, Rigaud AS. Designing robots for the elderly: appearance issue and beyond. Arch Gerontol Geriatr 2012; 54:121-6.

8. Hegel F, Lohse M, Wrede B. Effects of visual appearance on the attribution of applications in social robotics. Paper presented at: The 18th IEEE International Symposium on Robot and Human Interactive Communication; 2009 Sep 27-Oct 2; Toyama, Japan. Piscataway (NJ): IEEE; 2009. p. 64-71.

9. Prakash C, Kumar R, Mittal N. Recent developments in human gait research: parameters, approaches, applications, machine learning techniques, datasets and challenges. Artif Intell Rev 2018;49:1-40.

10. Kirkpatrick K. Legal issues with robots. Commun ACM 2013; 56:17-9. 